\section{Pseudotumor of the bile duct associated with autoimmune pancreatitis}

Autoimmune pancreatitis is a well-established entity that can be associated with biliary abnormalities [1]. Peroral cholangioscopic images of such biliary lesions are rarely available, however. We report here a patient with autoimmune pancreatitis who was found to have a pseudotumor in the bile duct.

A 69-year-old man with epigastric pain and general fatigue underwent transabdominal ultrasonography and computed tomography, which revealed a swollen pancreas and dilatation of the common bile duct. Though he did not have obstructive jaundice and had normal levels of gamma-immunoglobulin, endoscopic retrograde cholangiopancreatography (ERCP) showed that he had a stricture in the lower bile duct and an irregular main pancreatic duct (Figure 1). Although the results of the imaging studies were suspicious of autoimmune pancreatitis, the patient was observed without medication.

Eight months later he was hospitalized because he had developed obstructive jaundice. ERCP showed a polypoid lesion in upper bile duct, without a stricture of either the pancreatic duct or the distal bile duct (Figure 2). Peroral cholangioscopy revealed a tumor-like lesion with a thick vessel in the bile duct (Figure 3 ). The level of immunogloblin G subclass 4 (IgG4) was measured this time and was found to be high $(857 \mathrm{mg} / \mathrm{dl})$. A biopsy specimen from the lesion showed nonneoplastic bile-duct mucosa with fibrosis and lymphocyte and plasma cell infiltrations (Figure 4). We made a diagnosis of autoimmune pancreatitis with pseudotumor in the bile duct. The patient was treated with oral prednisolone $(40 \mathrm{mg} /$ day), and the jaundice improved immediately. Six months later, ERCP showed improvement in the narrowing of the bile duct, and peroral cholangioscopy revealed that the pseudotumor had disappeared. Three years after starting treatment, he is now on $5 \mathrm{mg}$ prednisolone daily.

Endoscopy_UCTN_Code_CCL_1AZ_2AC Endoscopy_UCTN_Code_TTT_1AO_2AI stricture in the lower bile duct. a stricture of the distal bile duct.

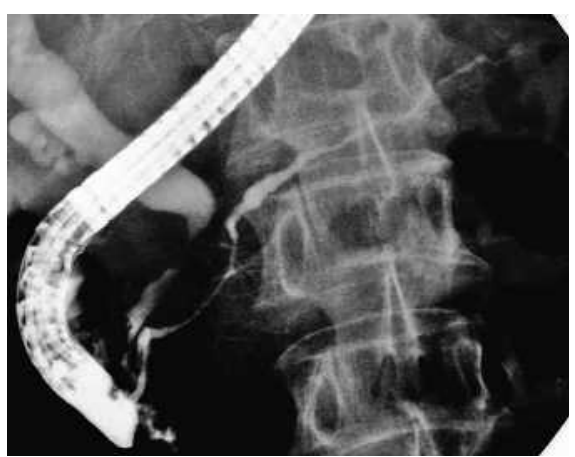

Figure 1 The initial endoscopic retrograde cholangiopancreatogram (ERCP) showed narrowing of the main pancreatic duct and a

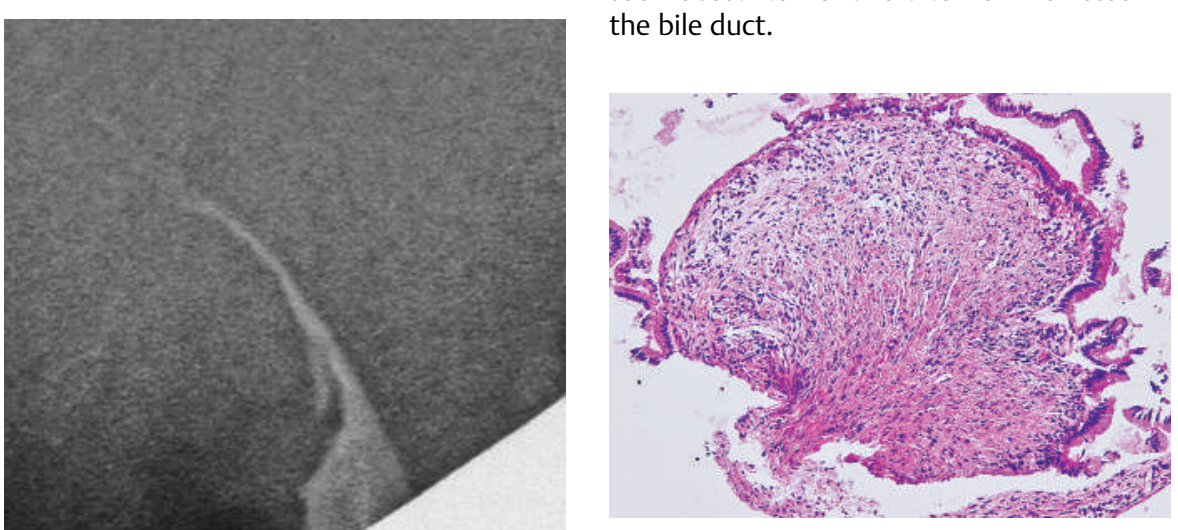

Figure 4 Histological examination of the biopsy specimens showed fibrosis with chronic inflammatory cell infiltrates (hematoxylin \& eosin stain, $\times 200$ magnification).

\section{References}

${ }^{1}$ Kamisawa T, Egawa N, Nakajima $\mathrm{H}$ et al. Clinical difficulties in the differentiation of autoimmune pancreatitis and pancreatic carcinoma. Am J Gastroenteol 2003; 98: 2694-2699

Corresponding author

\section{T. Itoi, M.D.}

Fourth Department of Internal Medicine Tokyo Medical University

Figure 2 On re-admission 8 months later, the patient underwent another ERCP, which showed an upper bile duct stricture without

\section{T. Kurihara, T. Itoi, A. Sofuni, F. Itokawa, T. Tsuchiya, F. Moriyasu}

Fourth Department of Internal Medicine, Tokyo Medical University, Tokyo, Japan.
Shinjuku-ku

Nishishinjuku 6-7-1

Tokyo

Japan

Fax: $\quad+81-3-5381-6654$

Email: itoi@tokyo-med.ac.jp

DOI: $10.1055 / s-2006-944597$ 